\title{
Interventions Following Traumatic Event in Children and Adolescents: An Evidence-Based Response
}

\author{
Saeed Farooq ${ }^{1 *}$, Muhammad Ayub $^{2}$ and Farooq Naeem
}

${ }^{1}$ Black Country NHS Foundation Trust, Postgraduate Medical Institute, Peshawar, Pakistan

${ }^{2}$ Department of Psychiatry Queen's University, Kingston Canada

${ }^{3}$ Queens University, Kingston, Ontario, Canada

\section{Introduction}

Recent attack on the Army Public School in Peshawar, Pakistan, is perhaps one the worst act of terrorism committed involving children and adolescent. There have been numerous acts of school shootings reported from Western and American schools but these have generally been acts of individuals [1]. Although this trauma is unique in many aspects, the region in which this school is located has borne the brunt of trauma and terror suffering for almost three decades now. Many school buildings have been destroyed in the region. A complex interplay of social, political and religious values, and witnessing violence as an everyday phenomenon already had an impact on the mental health of the population in general, and women and children in particular [2-4].

The mental health professionals are faced with a number of challenges in these situations. One major challenge is how we provide the evidence based treatments for such psychological trauma as well as prevent the long term consequences in children who are in the crucial stages of their emotional and cognitive development. These challenges are not easy to face especially when we consider lack of mental health services for children in places like Peshawar. The Mental Health Services for adults are already overstretched in providing the care for severe mental disorders. In this article we will provide the outline of an evidence based response in these situations.

\section{An Evidence-Based Response}

Faced with such a tragic incident the natural tendency is to act, rather than to think and act. People who are moved by the tragedy, with all the good will and noble intentions tend to rush in. However, this should not blind us from the need to base our response on the best available evidence. The need for the evidence-based response becomes particularly more relevant in the children where treatments can have long-term effects.

We suggested a framework for applying evidence based approach in similar situations, described in more details elsewhere $[5,6]$. The emphasis in this model is on adopting an approach guided by the best available evidence at every step. Following six steps, known by the acronym TRAUMA, would guide this approach

1. Training in interventions for which there is clear evidence of the effectiveness.

2. Research in the relevance and outcome of the interventions which we know can be effective but have limited evidence at present.

3. Apply the evidence-based interventions for disorders such as depression and anxiety as there are already a number of evidencebased treatments available for these disorders.

4. Understand the local context and limitations.

5. Modify the interventions in the light of the local context and limitations.
6. Apply again after the modification and understanding the local context as suggested in steps 4 and 5 above.

Following is a brief outline of this approach.

First step is the training in interventions for which there is clear evidence of the effectiveness. The major limiting factors for the application of these treatments in a developing country like Pakistan is the availability of trained professionals. While the mental health professionals can be trained to apply the interventions for which there is clear evidence of effectiveness, there would be many treatments which are not evaluated in the context of a developing Country. Therefore, it would be important to carry out the research in relevance and outcome of the intervention, as is suggested in the second step in this approach.

The interventions for terror-related trauma particularly in younger children and adolescent are relatively sparse in the developing countries. Therapies developed in Western cultures may be particularly difficult to apply in non western cultures due to important cultural, religious and social differences.

An important focus of the research should be to develop the screening instruments. When large populations are affected due to terror and trauma, it may not be possible to provide the effective treatments for all those affected or the interventions may not simply be effective (see below). These screening instruments can help to identify the children who are suffering from a psychiatric disorder and will be cost effective in utilising the meager resources for those who need these treatments the most.

It must be realized that the common psychiatric disorders such as anxiety, depression and substance abuse present the most formidable challenge in these populations. Third step in the TRAUMA approach requires that the effective treatment is provided for these disorders. Merikangas et al. [7] showed that approximately one in every four to five youth in the U.S. meets criteria for a mental disorder with severe impairment across their lifetime. Anxiety disorders were the most common condition (31.9\%), followed by behaviour disorders (19.1\%), mood disorders (14.3\%), and substance use disorders (11.4\%). The prevalence of these common psychiatric disorders is likely to be much higher in a population facing the humanitarian crisis. These

*Corresponding author: Saeed Farooq, Black Country NHS Foundation Trust Postgraduate Medical Institute, Peshawar, Pakistan, Tel: 905054037967; E-mail: sfarooqIrh@yahoo.com

Received February 13, 2015; Accepted March 10, 2015; Published March 17, 2015

Citation: Farooq S, Ayub M, Naeem F (2015) Interventions Following Traumatic Event in Children and Adolescents: An Evidence-Based Response: J Psychiatry 18: 269 doi:10.4172/psychiatry. 1000269

Copyright: $\odot 2015$ Farooq S, et al. This is an open-access article distributed under the terms of the Creative Commons Attribution License, which permits unrestricted use, distribution, and reproduction in any medium, provided the original author and source are credited 
disorders not only cause significant morbidity on their own but also act as predisposing and maintaining factors for the more severe forms of trauma-related psychiatric disorders in large populations. Almost all of this psychiatric morbidity either goes undetected and untreated. Therefore, the first challenge for any mental health service is to provide effective treatments for common mental disorders. Evidence-based interventions are available for the treatment of most of these disorders. These treatments should be provided on a priority basis.

Understanding the local context and limitations of our therapies and modifying the interventions in the light these, as required in step 4 and 5 is very important and goes beyond cultural understandings. The limitations posed by the ongoing conflict, difficult terrain, lack of trained professionals, inadequately developed road links, and local political sensitivities are often underestimated. One of the authors experienced this when an organization presented a plan for psychosocial support for those affected by the recent trauma in Army Public School in Peshawar. They consistently referred to Peshawar as the Ground Zero for all trauma-related activities in their strategy to address the psychosocial aspect of the trauma (meaning that the Peshawar will be the base for all activities being directly affected and the surrounding areas will follow). In a population that has been target of all the terror and trauma following the so called war on terror, this was a minor but obvious example of not understanding the local political sensitivities. The plan understandably did not impress the policy makers. The long distances that need to be covered, and the difficult terrains are important challenges which are being faced by the service providers in these areas. Innovative approaches such as Tele-Psychiatry can help in providing solutions for these problems.

An important limitation in applying these therapies is in understanding the role religion plays in the lives of those affected by the trauma. Religion is an important source of cultural identity and provides a framework and foundation on which both trauma and healing are interpreted. Unfortunately, in the current climate it has become difficult, even for the professionals to take an objective view of how the religion can be a significant factor in the healing process. A great majority of the population affected by trauma arising due to terrorism worldwide are Muslim. However, there is little systematic work done on how the religious identity of these populations affects the perception of trauma; and how religious beliefs can be incorporated in the therapy. Some studies have shown that this is possible. For example, Fernando et al. [8] found that among war-affected children in Sri Lanka, resilient orphans identified Buddhist religious practices such as meditation and reciting the five precepts of Buddhism as important coping strategies which promoted wellbeing. It is extremely important to understand how the religious identity affects the experience, and expression of mental distress in the context of present trauma, before any mental health interventions could be applied in these settings.

Finally, we will need to apply the interventions after modifications in the light of evidence and insights gained from the processes described above. Application in real-life settings not only helps the victims of torture and traumas, but it can also identify the gaps in our knowledge; practical problems in application; and the acceptability of interventions by local populations. Successful application should lead to satisfaction and a sense of achievement in health workers which is essential for sustaining those working in very difficult circumstances. If sufficient attention is not paid to the application of evidence based interventions in the field and learning from the practice in real World,, the entire process may become merely a theoretical exercise.

Application of the approach in a developing country context
It is not possible to elaborate the whole approach in the article, but will illustrate this by focusing on the first step, i.e., training and research in the interventions for which there is evidence of effectiveness. It would be interesting to examine the evidence for effectiveness of commonly adopted approaches and how these can be applied in a developing country setting.

The critical appraisal of two commonly adopted approaches in treatment of trauma faced by children and adolescent will show that two broad approaches are advocated for combating the effects of trauma which are discussed as below.

1. A universal approach targeting communities and schools delivered mostly by paraprofessionals. These interventions are delivered by non-specialist professionals to a broader group such as schools or classrooms.

2. The screen and treat approach: This approach is based on systematic screening of symptom and their severity at population level and then treatment is provided by highly trained professionals who would provide specialized treatments to those who are found to have symptoms meeting the criteria of a disorder or a predetermined severity.

\section{The Universal Approach}

The universal approach which targets groups and populations such as classrooms or schools appears to be cost effective way to dealing with the trauma considering that schools have been particular targets of the present violence in regions. School-based interventions vary in nature in different studies but usually consist of components such as psychoeducation, playful group cohesion activities and strengthening personal coping skills and in some programs, limited trauma-exposuren [9]. It seems that apparently 'non-invasive' interventions can help to fill the mental health service gaps in war-affected countries.

The Classroom-Based Intervention (CBI), a program that aims to reduce symptoms of PTSD, depression and anxiety and to increase children's resilience is a good example of a universal approach. CBI has been extensively studied in developing countries like Indonesia, Nepal, Sri Lanka [10-13]. Tol et al. aptly summarized the findings of these studies as following: 'Our current findings from Burundi add to a number of recent studies that have rigorously evaluated this schoolbased intervention with conflict-affected populations in Indonesia, Nepal, the occupied Palestinian territories, and Sri Lanka. Collectively, these studies provide emerging evidence-based answers to important questions on the practical benefit of this and similar interventions. First, given inconsistent results on primary outcome measures across settings, it now seems that this school-based intervention should not be recommended as a treatment for PTSD, depressive, and anxiety symptoms. Although the school-based intervention was associated with reductions in these symptoms in some settings (such as girls in Indonesia, boys and children with less ongoing trauma exposure in Sri Lanka, and children with both parents in Burundi), it was not associated with improvements in these symptoms in Nepal and the occupied Palestinian territories, and was associated with unfavourable effects on PTSD symptoms in Sri Lankan girls and older children in the occupied Palestinian territories [12].

A thought provoking commentary by Ertl and Neuner [9] accompanying this paper highlights the strengths of these studies in Low and Middle Income Countries (LAMIC) settings and notes that all four trials by the group of investigators [10-13] demonstrate that methodologically rigorous evaluation research can be implemented 
successfully in challenging contexts such as LAMIC Countries of limited political stability). They also point out that that universal prevention programs might interfere with the natural course of recovery and healthy readjustment of a minority of children. They conclude that 'It is tempting to perceive universal programs like $\mathrm{CBI}$ as a perfect solution to fill the mental health service gaps in war-affected countries, since they are low-threshold, cost-effective, resource-friendly and target many beneficiaries within a short period of time. However, the heterogeneity of beneficiaries and the lack of focus of the interventions have turned out to be problematic, as for some groups negative effects may occur ....... And CBI, and possibly also similar interventions should not be recommended as a treatment for symptoms of PTSD, depression and anxiety [9].

\section{The Screen and Treat Approach}

The systematic screening of symptom ensures that those who suffer from clinically relevant symptoms receive specialized treatment that, while those who recover on their own do not receive unnecessary treatment. It can be argued that the cost effectiveness of these individual therapies can be comparable to the universal approach if the trained lay therapists are employed with adequate supervision by clinical professionals and established referral pathways for more complex cases.

There are number of individual and group treatments which have shown the evidence of effectiveness. These include, for example, Trauma-Focused Cognitive-Behavioral Therapy (TF-CBT), and Narrative Exposure Therapy (NET) [14-16].

A recent systematic review [17] identified fourteen studies for adolescents exposed to different types of trauma such as sexual abuse, natural disaster, domestic violence and motor vehicle accidents. The psychological therapies used in these studies were cognitive behavioural therapy (CBT), exposure-based, psychodynamic, narrative, supportive counselling, and eye movement desensitisation and reprocessing (EMDR). Most studies compared a psychological therapy to a control group. Participants improved across all psychological therapies compared to the control group on measures such as PTSD (SMD $-0.90,95 \%$ CI -1.24 to -0.42 ), anxiety (three studies, $\mathrm{n}=91$, SMD -0.57 , 95\% CI -1.00 to -0.13 ) and depression (SMD $-0.74,95 \%$ CI -1.11 to -0.36). The best evidence amongst the psychological therapies was for CBT. Improvement was significantly better for up to a year following treatment (up to one month: two studies, $n=49$, OR $8.64,95 \%$ CI 2.01 to 37.14; up to one year: one study, $\mathrm{n}=25$, OR $8.00,95 \%$ CI 1.21 to 52.69 ).

\section{Conclusion}

It is important that we apply those interventions that work; and not those which seem to work. In resource poor setting evidence based approach is the basic need, not a luxury. The mental health consequences of the trauma in children pose significant humanitarian crisis. The knee-jerk response in these situations results in wasting the valuable resources and exposing the children to the risks which can't be quantified in the present state of knowledge. The evaluation of CBI as explained above is an excellent example of this. It seems that those interventions which have sound theoretical underpinning such as cognitive theory have better evidence of effectiveness. The challenge for mental health professionals in developing countries is to modify these interventions in the light of local context and limitations.

Citation: Farooq S, Ayub M, Naeem F (2015) Interventions Following Traumatic Event in Children and Adolescents: An Evidence-Based Response: J Psychiatry 18: 269 doi:10.4172/psychiatry. 1000269
The whole process may seem too tedious and perhaps not feasible. It is however, not necessary to begin with all the steps simultaneously. Searching and generating evidence should go hand-in-hand with its application. Even if it is not possible to apply the treatments for trauma related interventions, it would be possible to apply the evidence-based interventions for the mental disorders such as anxiety and depression, which are readily available. The emphasis in this approach is on systematic applications of evidence based solutions. If the relevant evidence is lacking or not applicable in a particular situation, it is important that we are aware of the gap in the knowledge and hence the limitation of the treatment we are using.

\section{References}

1. Metzl JM, MacLeish KT (2015) Mental illness, mass shootings, and the politics of American firearms. Am J Public Health 105: 240-249.

2. Ayub M, Irfan M, Nasr T, Lutufullah M, Kingdon D, et al. (2009) Psychiatric morbidity and domestic violence: a survey of married women in Lahore. Soc Psychiatry Psychiatr Epidemiol 44: 953-960.

3. Ayub M, Poongan I, Masood K, Gul H, Ali M, et al. (2011) Psychological Morbidity in Children 18 months after Kashmir Earthquake of 2005.Child Psychiatry Hum Dev.

4. Naeem F, Ayub M, Masood K, Gul H, Khalid M, et al. (2011) Prevalence and psychosocial risk factors of PTSD: 18 months after Kashmir earthquake in Pakistan. J Affect Disord 130: 268-274.

5. Farooq S (2005) Knowledge is the enemy of disease: an evidence based approach to the earthquake in Pakistan. J Pak Pyschiatric Soc 2: 57-58.

6. Farooq S (2006) Mental health research agenda for the 2005 earthquake in Pakistan. J Coll Physicians Surg--Pak JCPSP 16: 85-86.

7. Merikangas KR, He J-P, Burstein M, Swanson SA, Avenevoli S, et al. (2010) Lifetime prevalence of mental disorders in U.S. adolescents: results from the National Comorbidity Survey Replication--Adolescent Supplement (NCS-A). J Am Acad Child Adolesc Psychiatry 49: 980-989.

8. Fernando C (2006) Children of war in Sri Lanka: Promoting resilience through faith development. ProQuest.

9. Ertl V, Neuner F (2014) Are school-based mental health interventions for waraffected children effective and harmless? BMC Med 12: 84

10. Tol WA, Komproe IH, Susanty D, Jordans MJ, Macy RD, et al. (2008) Schoolbased mental health intervention for children affected by political violence in Indonesia: a cluster randomized trial. JAMA 300: 655-662.

11. Tol WA, Komproe IH, Jordans MJD, Vallipuram A, Sipsma H, et al. (2012) Outcomes and moderators of a preventive school-based mental health intervention for children affected by war in Sri Lanka: a cluster randomized trial. World Psychiatry 11: 114-122.

12. Tol WA, Komproe IH, Jordans MJD, Ndayisaba A, Ntamutumba P, et al. (2014) School-based mental health intervention for children in war-affected Burundi: a cluster randomized trial. BMC Med 12: 56

13. Jordans MJD, Komproe IH, Tol WA, Kohrt BA, Luitel NP, et al. (2010) Evaluation of a classroom-based psychosocial intervention in conflict-affected Nepal: a cluster randomized controlled trial. J Child Psychol Psychiatry 51: 818-826.

14. Robjant K, Fazel M (2010) The emerging evidence for Narrative Exposure Therapy: a review. Clin Psychol Rev 30: 1030-1039.

15. O'Callaghan P, McMullen J, Shannon C, Rafferty H, Black (2013) A randomized controlled trial of trauma-focused cognitive behavioral therapy for sexually exploited, war-affected Congolese girls. J Am Acad Child Adolesc Psychiatry 52: 359-369.

16. Catani C, Kohiladevy M, Ruf M, Schauer E, Elbert T, et al. (2009) Treating children traumatized by war and Tsunami: a comparison between exposure therapy and meditation-relaxation in North-East Sri Lanka. BMC Psychiatry 9: 22.

17. Gillies D, Taylor F, Gray C, O'Brien L, D'Abrew N (2012) Psychological therapies for the treatment of post-traumatic stress disorder in children and adolescents Cochrane Database of Systematic Reviews, Issue 12. Art. No: CD006726. 